\title{
Nicht alle niedermolekularen Heparine sind gleich
}

- Wegen seiner pharmakologischen Besonderheiten im Vergleich zu anderen niedermolekularen Heparinen (NMH) kann Tinzaparin (innohep ${ }^{\circledR}$ ) auch bei Patienten mit eingeschränkter Nierenfunktion oder Tumorerkrankungen in voller therapeutischer Dosierung eingesetzt werden und ermöglicht so eine effektive Rezidivprophylaxe.

Eine therapeutische Antikoagulation kann sich bei geriatrischen Patienten als Herausforderung erweisen: Einschränkungen der Nierenfunktion, herabgesetzte Homöostaseregulation, Multimorbidität und Mehrfachtherapie können die Therapie deutlich erschweren, wie Prof. Martin Wehling, Mannheim, zu bedenken gab. Zugleich steige das Blutungsrisiko unter Kumarinen mit dem Alter an.

\section{(Keine) Akkumulationsgefahr bei} abnehmender Nierenfunktion

$\mathrm{NMH}$ haben zwar ein niedrigeres Blutungsrisiko als Vitamin-K-Antagonisten, dennoch sollte besonders bei niereninsuffizienten Patienten sorgfältig auf die Unterschiede zwischen den Präparaten geachtet werden. Die verfügbaren NMH unterscheiden sich in ihrer chemischen Struktur. Längere Halbwertszeiten und kurze Kettenlängen erhöhen das Risiko für eine Akkumulation bei abnehmender Nierenfunktion (Kreatinin-Clearance $<60$ $\mathrm{ml} / \mathrm{min}$ ). Eine Dosisreduktion berge aber die Gefahr einer Abschwächung der therapeutischen Wirkung, so Wehling. Tinzaparin biete den Vorteil, dass bis zu einer Kreatinin-Clearance von $\geq 20 \mathrm{ml} / \mathrm{min}$ keine Dosisanpassung erforderlich sei. So- mit könnten Patienten mit eingeschränkter Nierenfunktion sowie Tumorpatienten mit der vollen therapeutischen Dosis behandelt werden.

Tinzaparin weist im Vergleich zu anderen NMH einen größeren Anteil an längeren Heparinketten und ein höheres Molekulargewicht auf, erläuterte Prof. Susanne Alban, Kiel. Während NMH mit geringerem Molekulargewicht überwiegend renal eliminiert werden und bei Nierenfunktionseinschränkungen somit das Blutungsrisiko erhöhen können, wird Tinzaparin unabhängig von der Nierenfunktion metabolisiert.

- Abdol A. Ameri

Quelle: Satellitensymposium, DACH-Tagung Angiologie, Basel, 13. September 2010 (Veranstalter: LEO Pharma)

\section{Mängel bei der präoperativen Diagnostik \\ In Deutschland werden zu viele Schilddrüsen operiert}

- Die Zahl der jährlichen Schilddrüsenoperationen in Deutschland ist mit rund 120000 eindeutig zu hoch. Dies ist vor allem der Tatsache geschuldet, dass bei asymptomatischen kleinen Strumen mit solitären oder multiplen Knoten der Verdacht auf ein Schilddrüsenmalignom präoperativ nicht ausgeräumt wird, so Prof. Rainer Hehrmann, Hannover. Durch eine verbesserte Diagnostik mittels differenzierter Ultraschallkriterien und Feinnadelpunktion könnten viele unnötige Operationen vermieden werden.

Nur bei drei von 10000 Schilddrüsenknoten handelt es sich um ein Karzinom. Die hohe Prävalenz benigner Strumen und Schilddrüsenknoten ist in Deutschland durch Jodmangel zu erklären. Die Ausbildung von Schilddrüsenautonomien, in Deutschland die häufigste Ursache einer Hyperthyreose, ist ebenfalls auf den Jodmangel zurückzuführen, so Prof. Petra-Maria Schumm-Dräger, München. Eine ausreichende Jodversorgung und eine suffiziente Therapie der Struma diffu-

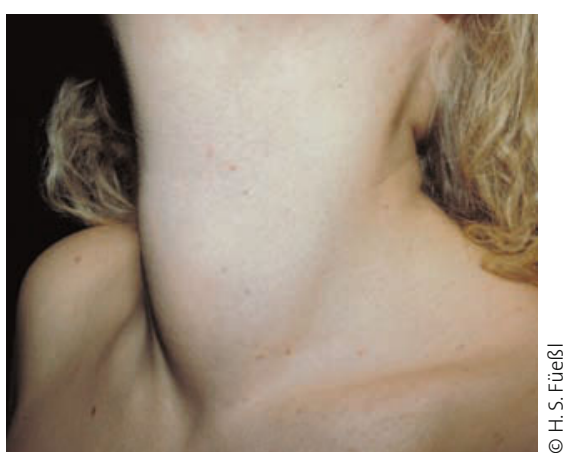

gestrebt werden. Bei einer Hyperthyreose vom Typ M. Basedow werde dagegen eine mindestens einjährige Thyreostatikatherapie empfohlen, da es in 40-70\% der Fälle innerhalb dieser Zeit zu einer Spontanremission komme.

- Verena Horsthemke

Quelle: Schilddrüsensymposium 2010, München, 14. Juli 2010 (Veranstalter: Merck Serono)

Struma nodosa bei autonomen Adenom.

sa mit Levothyroxin/Jod stelle die beste Prophylaxe einer Hyperthyreose dar. Auch bei Schilddrüsenknoten und Struma nodosa sei die Kombinationstherapie mit Einstellung des TSH-Wertes in den unteren Normbereich erfolgreicher als eine Monotherapie mit Jodid oder Levothyroxin.

Bei Schilddrüsenautonomie mit subklinischer oder manifester Hyperthyreose sollte immer eine definitive Therapie wie eine Radiojodtherapie oder Operation an-

\section{Krebsrisiko durch Radiojod?}

Die Radiojodtherapie stellt bei Schilddrüsenautonomien, $M$. Basedow oder Schilddrüsenmalignomen eine effektive und nebenwirkungsarme Therapieoption dar, erklärt der Nuklearmediziner Prof. Martin Luster, Ulm. Die Sorge, durch die Strahlenbelastung die Entstehung von Malignomen zu fördern, sei häufig unbegründet. Bei mehr als 60-jähriger Anwendung der Radiojodtherapie ließen sich keine eindeutigen Nachweise für ein gesteigertes posttherapeutisches Malignomrisiko finden. 